AUTORES:

Daniel Duarte ${ }^{1}$

Júlio Garganta ${ }^{2}$

António Manuel Fonseca ${ }^{2}$

${ }^{1}$ ISMAI, Portugal

${ }^{2} \mathrm{CIFI}^{2} \mathrm{D}$, Faculdade de Desporto Universidade do Porto, Portugal

https://doi.org/10.5628/rpcd.12.03.12

\section{Adaptação transcultural}

e avaliação das propriedades

psicométricas e estrutura

factorial da versão

portuguesa da Coaching

Efficacy Scale (CESp).

RESUMO

Da análise da literatura da especialidade, resulta que a Coaching Efficacy Scale (CES) se tem constituído como o principal instrumento utilizado pelos investigadores para avaliar a eficácia dos treinadores desportivos. Assim, o objectivo deste estudo consistiu em proceder à sua tradução e adaptação transcultural para a realidade portuguesa, bem como à avaliação da qualidade das propriedades psicométricas e da estrutura factorial da versão resultante (i.e., da CESp). Participaram 244 futebolistas seniores masculinos de clubes participantes na Primeira e na Segunda Liga Portuguesa de Futebol Profissional, com idades compreendidas entre os 18 e os 35 anos de idade. De uma forma geral, a análise dos resultados relativos às matrizes de correlações inter-item, item-total e inter-factor e aos valores dos alfas de Cronbach evidenciou a fiabilidade da CESp. Adicionalmente, as AFC revelaram que os modelos de medida unidimensional e multidimensional (constituído por quatro factores idênticos aos propostos pelos autores da versão original e reforçados em vários estudos posteriores) apresentaram um ajustamento aceitável à matriz de covariância dos dados empíricos recolhidos, sugerindo, portanto, que pode ser usada com confiança tanto como uma medida global para a avaliação da eficácia dos treinadores como para avaliar individualmente cada uma das quatro facetas nela representadas. Nesse sentido, recomenda-se pois a sua utilização por parte dos investigadores nacionais interessados nesta área do conhecimento. 
of the psychometric properties

and factorial structure of the Portuguese version

of the Coaching Efficacy Scale (CESp).

\section{ABSTRACT}

The analysis of the literature reveals that the Coaching Efficacy Scale (CES) is the main tool used by researchers to assess the efficacy of sports coaches. Thus, the aim of this study was to undertake the translation and transcultural adaptation to Portuguese reality, as well as the assessment of the quality of the psychometric properties and factor structure of the resulting version (ie, CESp). Participated 244 male football players, aged between 18 and 35 years old, from clubs participating in the First and Second Portuguese Professional Football League. In general, the analysis of the results of inter-item, item-total and inter-factor matrix correlations, and of the values of Cronbach's alphas, showed the reliability of the CESp. Additionally, the CFA revealed that the single-dimensional and multidimensional (consisting of four factors identical to those proposed by the authors of the original version and reinforced in several subsequent studies) measurement models showed an acceptable adjustment to the covariance matrix of the empirical data collected, suggesting therefore it can be safely used both as a measure for the overall evaluation of the coach efficacy as to individually evaluate each of the four faces shown therein. Therefore, it is recommended its use by national researchers interested in this area of knowledge.

\section{KEY WORDS:}

Coaching. Efficacy. Coaching Efficacy Scale. Cross-cultural adaptation. Psychometric properties. Factorial structure. 


\section{INTRODUÇÃo}

0 treinador apresenta-se como um dos principais intervenientes do processo desportivo, existindo um consenso relativo à importância da sua função enquanto potenciador dos níveis de rendimento ou performance dos atletas ${ }^{(6,30,38)}$, bem como à necessidade de desenvolver um vasto reportório de competências técnicas e psicológicas que the permita desempenhar as suas funções com o sucesso desejado ${ }^{(4,22 ; 43)}$.

Porém, a obtenção e a avaliação do sucesso desportivo do treinador constituem-se como tarefas difíceis, envolvendo vários critérios e variáveis de natureza subjectiva, de entre as quais se poderão destacar as relativas à sua eficácia, seja esta considerada de forma mais objectiva ou subjectiva, designadamente no plano das expectativas.

$\mathrm{Na}$ linha de Bandura ${ }^{(1)}$, as expectativas de auto-eficácia do treinador podem ser entendidas como os julgamentos produzidos por ele sobre a sua capacidade para influenciar o processo de aprendizagem e o rendimento dos seus atletas. Sendo determinadas essencialmente pelas suas realizações anteriores, experiências vicariantes, persuasão verbal e estados fisiológicos, as expectativas dos treinadores determinam em grande medida o tipo de comportamentos que adoptam, bem como a sua intensidade e persistência, constituindo-se portanto como preditoras do seu sucesso e, consequentemente, do sucesso dos respectivos atletas e equipas.

$\mathrm{Na}$ verdade, de acordo com o modelo proposto por Horn ${ }^{(21)}$ sobre a efectividade do treinador, os comportamentos dos treinadores são influenciados pelos seus objectivos, crenças e expectativas, os quais são por sua vez influenciados por outras variáveis relacionadas, por exemplo, com as características dos próprios treinadores, mas também dos seus atletas e dos contextos em que se desenrola o processo desportivo. Do mesmo modo, também a efectividade dos treinadores é condicionada por factores que thes são intrínsecos bem como por outros de natureza situacional.

Sendo a efectividade entendida como o período de tempo em que os treinadores conseguem aplicar o seu conhecimento e competências no sentido de influenciar positivamente a performance e a aprendizagem dos seus atletas, relacionando-se com uma maior satisfação, auto-estima e percepção da realidade por parte destes ${ }^{(21)}$, parece ser evidente que uma das suas determinantes é a eficácia do treinador ${ }^{(16,36)}$.

A eficácia do treinador constitui-se pois como um factor determinante na constância com que o treinador adopta os comportamentos mais ajustados aos recursos disponíveis e consegue contribuir para a obtenção dos mais elevados rendimentos possíveis ${ }^{(30)}$.

No que se refere à avaliação da eficácia do treinador, a análise da literatura disponível neste domínio destaca inequivocamente o relevo assumido pela Coaching Efficacy Scale (CES) ${ }^{(15)}$, referida mesmo como sendo o único instrumento publicado para este efeito ${ }^{(34,36)}$.

Baseando-se no trabalho preliminar de Park ${ }^{(37)}$ acerca da confiança do treinador, no conceito de auto-eficácia de Bandura (1) e no modelo multidimensional de ensino eficaz de 
Denham e Michael ${ }^{(13)}$, Feltz e colaboradores ${ }^{(15)}$ desenvolveram a CES com o objectivo de criar um instrumento que permitisse avaliar a eficácia do treinador, considerando-a em função do seu nível de confiança no desempenho bem sucedido de várias tarefas específicas da sua actividade profissional; de sublinhar que, na linha do anteriormente referido por Feltz e Chase ${ }^{(14)}$ e Myers, Feltz, Chase, Reckase e Hancock ${ }^{(34)}$ sobre a possibilidade de a auto-eficácia poder ser entendida como a autoconfiança face a uma situação específica, os termos eficácia e confiança são utilizados de forma similar ao longo deste artigo.

A versão final da CES é constituída por 24 itens correspondentes cada um deles a um indicador de eficácia específica do treinador, os quais se agrupam em quatro dimensões mais abrangentes: i) 'motivação' (i.e., confiança na capacidade para influenciar as competências e estados psicológicos dos seus atletas e equipas); ii) 'estratégia de jogo' (i.e., confiança na capacidade para promover uma performance de sucesso dos seus atletas e equipas durante a competição); iii) 'técnica' (i.e., confiança na capacidade para diagnosticar e desenvolver as competências dos seus atletas e equipas durante o processo de treino); e iv) 'construção do carácter' (i.e., confiança na capacidade para influenciar o desenvolvimento pessoal e uma atitude positiva dos seus atletas e equipas perante o desporto).

Desde a sua publicação, a CES tem vindo a ser escolhida por um conjunto alargado de investigadores (e.g., 9, 15, 33, 39, 42) para analisar o modo como os treinadores avaliam a sua eficácia, suas fontes e consequências, ou ainda o padrão de relações estabelecido com outras variáveis. Adicionalmente, vários estudos em que foram analisadas as propriedades psicométricas da CES também têm demonstrado a sua qualidade e utilidade (e.g., 3, 18, 36, 39, 41), justificando-se portanto a sua tradução e adaptação transcultural para contextos distintos da realidade norte-americana, procurando assim contribuir para a promoção da investigação e do aumento do conhecimento nesta área.

Na realidade, a CES tem sido utilizada predominantemente em estudos realizados em países anglo-saxónicos $(3,8,15,16,18,26,27,33,36,40)$ sendo extremamente escassa a investigação desenvolvida noutros países, designadamente em Portugal e nos restantes países lusófonos.

Para além disso, em grande medida relacionada com a questão anterior, não pode deixar de se estranhar que seja praticamente inexistente a investigação realizada no contexto do futebol, sendo este um dos desportos mais praticados a nível mundial e em que os treinadores desempenham um papel tão importante e decisivo. Com efeito, nesta área, apenas é possível identificar na literatura um estudo exclusivamente desenvolvido com treinadores de futebol (27), ao qual se poderá acrescentar alguma informação proveniente de um escasso número de outros estudos em que também participaram treinadores de futebol mas juntamente com treinadores de outras modalidades colectivas (e.g., 3,15, 16, 26), justificando-se portanto o aprofundamento do conhecimento actualmente disponível neste domínio.

Procurando igualmente contribuir para o aumento do conhecimento nesta área, recentemente, Myers, Feltz, Maier, Wolfe e Reckase ${ }^{(35)}$ decidiram utilizar a CES para avaliar as 
percepções de um conjunto de atletas de equipas masculinas e femininas de hóquei no gelo e de futebol relativamente aos seus treinadores através da modificação das instruções iniciais: em lugar de pedirem aos treinadores para avaliarem a sua eficácia (recorrendo à questão inicial: "Que confiança sente na sua capacidade para...", complementada depois pelos 24 itens que integram a CES, cada um deles relativo a um comportamento específico do treinador), foi pedido aos atletas para avaliarem a competência demonstrada pelos seus treinadores (recorrendo à questão inicial "Qual é a competência do teu treinador para...", complementada depois pelos mesmos 24 itens que integram a CES).

Não sendo inédito o procedimento de utilizar um mesmo elenco de itens para avaliar construtos relativamente distintos, modificando para o efeito as instruções iniciais (e.g. $10,17)$, este estudo, ao fornecer evidências no sentido da validade desta versão modificada da CES (que os autores optaram por designar como Coaching Competence Scale), veio demonstrar a viabilidade da adopção desse procedimento para a CES e suscitar a sua expansão a outras possibilidades.

Por exemplo, conforme destaca Hedstrom (20), apesar de ser conhecido o impacto que perspectivas divergentes poderão assumir no clima e no rendimento das respectivas equipas, a investigação produzida sobre as semelhanças e diferenças existentes entre o modo como treinadores e atletas percebem a eficácia dos comportamentos dos primeiros é reduzida, razão pela qual importa incrementá-la. $E$, na mesma linha, parece ser igualmente relevante perceber em que medida convergem, ou não, treinadores e atletas na valorização do contributo de determinados comportamentos para a obtenção dos mais elevados índices de sucesso, porquanto, se existirem diferenças significativas a este nível e sabendo-se do modo como a adopção de determinados comportamentos é condicionada pelas crenças relativamente às vantagens que daí decorrerão para os próprios, será naturalmente necessário intervir nesse sentido.

Uma outra questão que tem reclamado atenção por parte dos investigadores é a que se refere à estrutura factorial da CES, porquanto Feltz e colaboradores ${ }^{(15)}$, apesar de a terem desenvolvido com o objectivo de testar a multidimensionalidade da eficácia do treinador proposta no seu modelo teórico e de terem fornecido evidências no sentido da validade da estrutura com os quatros factores anteriormente referidos, sublinharam igualmente que os seus resultados proporcionaram suporte marginal para a existência de apenas um factor único de eficácia.

Posteriormente, os resultados de um estudo realizado por Myers e colaboradores (36) evidenciaram novamente as boas propriedades psicométricas da CES e forneceram apoio para o entendimento da eficácia desportiva como um construto multidimensional (constituído pelos quatro factores propostos: motivação, estratégia de jogo, técnica e construção do carácter), mas não para a existência de um factor unidimensional mais abrangente. No mesmo sentido, também Tsorbatzudis, Daroglou, Zahariadis e Grouious ${ }^{(41)}$, ao avaliarem 
a versão grega da CES, encontraram suporte para a utilização da escala multifactorial. Ainda assim, a dúvida anteriormente colocada parece permanecer, pois outros autores (e.g."

39), independentemente de sublinharem a existência de apoio para a estrutura com quatro factores, têm reportado igualmente resultados satisfatórios com a utilização de apenas um factor de eficácia total.

Em conformidade com o anteriormente exposto, constituiu-se como principal objectivo deste trabalho proceder à tradução e adaptação da CES para a realidade portuguesa e à verificação das suas propriedades psicométricas e da estrutura factorial mais adequada, quando aplicada no contexto do futebol, designadamente com atletas. Pretendeu-se assim disponibilizar aos investigadores nacionais um instrumento de reconhecida qualidade que permita desenvolver a investigação neste domínio em Portugal, mas também contribuir para a testagem do modelo conceptual que a suporta, proposto por Feltz e colaboradores ${ }^{(15)}$, ao expandir a investigação a contextos e culturas distintos daqueles em que tem vindo a ser aplicado.

\section{METODOLOGIA}

AMOSTRA

Considerando os propósitos do estudo (que implicavam que os respondentes possuíssem um conhecimento suficientemente substantivo e sustentado sobre as questões em análise; i.e., sobre a importância de determinados comportamentos dos treinadores para a obtenção de elevados índices de rendimento desportivo no futebol), entendeu-se recorrer a uma amostra constituída apenas por atletas com experiência de elevado nível competitivo.

Assim, a amostra foi composta por 244 futebolistas seniores masculinos de clubes participantes nos campeonatos nacionais da Primeira Liga e da Segunda Liga Portuguesas de Futebol Profissional, com idades compreendidas entre os 18 e os 35 anos de idade.

\section{INSTRUMENTO}

Conforme referido anteriormente, a CES foi desenvolvida por Feltz e colaboradores ${ }^{(15)} \mathrm{e}$ é constituída por 24 itens distribuídos por quatro factores: i) 'motivação'; ii) 'estratégia de jogo'; iii) 'técnica'; e iv) 'construção do carácter', relativamente aos quais se responde através de uma escala de Likert. Embora no estudo original tenha sido proposta uma escala de resposta de 10 pontos (de $0=$ nada confiante a $10=$ extremamente confiante), na maior parte dos estudos que se seguiram tem vindo a ser utilizada uma escala de apenas 5 pontos (36). Nesse sentido, e porque um número elevado de opções de resposta pode levar a uma maior dificuldade em distinguir entre elas e diminuir a validade dos dados recolhidos ${ }^{(12)}$, neste estudo optou-se igualmente pela escala de resposta com 5 pontos. 


\section{PROCEDIMENTOS}

Os propósitos do estudo foram previamente comunicados aos atletas, aos quais foi igualmente garantida a confidencialidade das respostas e esclarecido que não se pretendia proceder a qualquer tipo de avaliação dos seus treinadores, mas sim conhecer o modo como consideravam que cada um dos comportamentos dos treinadores indicados na CES contribui, ou não, para a obtenção dos mais elevados níveis de rendimento, não existindo por isso mesmo respostas 'certas' ou 'erradas'.

A recolha de dados, que se processou em 10-15 minutos, foi conduzida pelo primeiro autor deste trabalho, maioritariamente, nas instalações dos clubes dos atletas inquiridos; nos casos em que isso não foi possível, porquanto as respectivas equipas se encontravam em estágio, a recolha dos dados processou-se nos locais dos estágios.

\section{ANÁLISE DOS DADOS}

A versão original da CES foi traduzida da língua inglesa para a língua portuguesa por dois especialistas bilingues, tendo a versão resultante (designada de CESp) sido posteriormente submetida à apreciação de um júri, constituído por 5 elementos com domínio de ambas as línguas e conhecimento aprofundado na área da psicologia do desporto, que comparou ambas as versões e concluiu no sentido da existência de equivalência em termos de semântica e de conteúdo.

Posteriormente, foram realizadas várias sessões de reflexão falada com um conjunto de 10 treinadores e atletas com o objectivo de determinar a compreensibilidade e uniformidade intercontextual da CESp. No decurso destas sessões foi possível identificar a existência de algumas dificuldades na interpretação de dois itens, bem como da questão inicial e da escala de resposta, pelo que se entendeu como conveniente proceder a ligeiras modificações. Mais especificamente, os itens “... demonstrar as competências específicas do futebol" e "... tomar decisões críticas durante a competição" foram reformulados para “...demonstrar as competências do futebol" e “... tomar decisões importantes durante a competição", respectivamente. Do mesmo modo, a questão inicial "Na sua opinião, para um treinador de futebol ser bem sucedido, que eficácia terá que ter em relação a cada um dos seguintes comportamentos..." foi reformulada para "Na sua opinião, qual é a importância de cada um dos seguintes comportamentos para um treinador ser bem sucedido..." e a escala de resposta, em lugar de referir-se a eficácia (i.e., de variar entre 1 =nada eficaz a $5=$ muito eficaz) passou a referir-se a importância (i.e., passou a variar de 1 =nada importante a 5 =totalmente importante).

A análise estatística dos dados recolhidos foi efectuada através do recurso ao PASW (Predictive Analytics SoftWare) 18.00 e ao LISREL 8.54 for Windows e iniciou-se pela análise exploratória dos dados obtidos, concretamente no que se refere ao cálculo da média, desvio-padrão, assimetria e achatamento de cada um dos 24 itens da CESp, seguida pela 
avaliação da consistência interna de cada um dos seus quatro factores e da escala total, através do cálculo dos respectivos valores de alfa de Cronbach, e pela análise das correlações inter-item e item-total, relativamente a cada factor e à escala total, e das correlações inter-factores e factor-total.

No que se refere à estrutura factorial da CESp, considerando as indicações disponíveis na literatura a este respeito a que anteriormente nos referimos, decidiu-se avaliar o ajustamento da estrutura multifactorial (com os quatro factores propostos pelos autores) à matriz de dados recolhidos neste estudo, mas também o ajustamento desta matriz a um modelo com apenas um factor. Para o efeito, foi utilizada a técnica da Análise Factorial Confirmatória (AFC), tendo sido selecionados para o efeito os indicadores mais frequentemente referenciados pela literatura internacional neste domínio: $\chi^{2}$, rácio entre $0 \chi^{2}$ e os graus de liberdade $\left(\chi^{2} / \mathrm{gl}\right)$, Standardized Root Mean Square Residual (SRMR), Root Mean Square Error of Approximation (RMSEA), Non-Normed Fit Index (NNFI) e Comparative Fit Index (CFI).

\section{RESULTADOS E DISCUSSÃO}

Através da consulta ao quadro 1 é possível verificar que, no seu conjunto, os atletas não utilizaram todas as possibilidades de resposta em relação a alguns dos comportamentos dos treinadores indicados na CESp (nomeadamente as mais baixas; i.e., correspondentes a uma avaliação de 'nada importante' ou 'pouco importante') e que os valores médios foram todos superiores a 4, o que é perfeitamente compreensível se se atender ao facto de todos os comportamentos indicados serem orientados para a procura do aumento do rendimento e do sucesso dos respectivos atletas ou equipas. Quanto à normalidade da distribuição dos valores das respostas foi possível verificar a inexistência de fenómenos de assimetria e achatamento.

QUADRO 1 - Valores mínimos (mín), máximos (máx), médios (M), dos desvios padrão

(dp), de assimetria (ass) e achatamento (ach) das respostas aos itens da CESp.

\begin{tabular}{lcclll}
\hline ITEM & MÍN-MÁx & M & DP & ASS & ACH \\
\hline CESp.01 & $3-5$ & 4.55 & 0.60 & -0.99 & -0.02 \\
\hline CESp.02 & $2-5$ & 4.14 & 0.73 & -0.45 & -0.30 \\
\hline CESp.03 & $2-5$ & 4.34 & 0.67 & -0.66 & 0.00 \\
\hline CESp.04 & $2-5$ & 4.29 & 0.69 & -0.59 & -0.22 \\
\hline CESp.05 & $2-5$ & 4.33 & 0.66 & -0.56 & -0.35 \\
\hline CESp.06 & $1-5$ & 4.40 & 0.64 & -0.93 & 1.77 \\
\hline CESp.07 & $1-5$ & 4.33 & 0.74 & -0.96 & 0.94 \\
\hline
\end{tabular}




\begin{tabular}{|c|c|c|c|c|c|}
\hline ITEM & MÍN-MÁX & $M$ & $\mathrm{DP}$ & ASS & $\mathrm{ACH}$ \\
\hline CESp.08 & $2-5$ & 4.40 & 0.67 & -0.89 & 0.55 \\
\hline CESp.09 & $2-5$ & 4.16 & 0.72 & -0.53 & 0.02 \\
\hline CESp.10 & $2-5$ & 4.62 & 0.58 & -1.50 & 1.38 \\
\hline CESp.11 & $2-5$ & 4.34 & 0.61 & -0.46 & 1.38 \\
\hline CESp.12 & $1-5$ & 4.38 & 0.62 & -0.85 & 1.91 \\
\hline CESp.13 & $2-5$ & 4.34 & 0.78 & -1.00 & 0.37 \\
\hline CESp.14 & $2-5$ & 4.06 & 0.74 & -0.37 & -0.33 \\
\hline CESp.15 & $2-5$ & 4.40 & 0.63 & -0.75 & 0.39 \\
\hline CESp.16 & $2-5$ & 4.39 & 0.62 & -0.60 & -0.09 \\
\hline CESp.17 & $1-5$ & 4.26 & 0.67 & -0.73 & 1.33 \\
\hline CESp.18 & $1-5$ & 4.28 & 0.71 & -0.89 & 1.30 \\
\hline CESp.19 & $2-5$ & 4.33 & 0.72 & -0.65 & -0.57 \\
\hline CESp.20 & $1-5$ & 4.33 & 0.70 & -0.94 & 1.38 \\
\hline CESp.21 & $2-5$ & 4.34 & 0.69 & -0.77 & 0.27 \\
\hline CESp.22 & $3-5$ & 4.30 & 0.66 & -0.42 & -0.74 \\
\hline CESp.23 & $2-5$ & 4.54 & 0.59 & -0.99 & 0.54 \\
\hline CESp.24 & $2-5$ & 4.43 & 0.67 & -0.90 & 0.30 \\
\hline
\end{tabular}

Quanto à consistência interna dos quatro factores da CESp, a análise dos valores de alfa de Cronbach revelou que eram todos superiores a 0.70 (ver quadro 2), não sendo passiveis de aumento a partir da eliminação de qualquer dos seus itens. Quando considerado apenas um factor único o coeficiente de alfa foi substancialmente superior, atingindo o valor de 0.91.

QUADRO 2 - Valores das correlações inter-item, em cada factor, item-factor e item-total e dos alfas de Cronbach relativos aos quatro factores da CESp.

\begin{tabular}{|c|c|c|c|c|}
\hline ITEM-FACTOR & INTER-ITEM & ITEM-FACTOR & ITEM-TOTAL & ALFA \\
\hline motivação & $0.10-0.49$ & & & 0.71 \\
\hline CESp.01 & $0.10-0.34$ & 0.36 & 0.35 & \\
\hline CESp.03 & $0.07-0.30$ & 0.29 & 0.40 & \\
\hline CESp.06 & $0.07-0.35$ & 0.39 & 0.35 & \\
\hline CESp.10 & $0.10-0.40$ & 0.42 & 0.41 & \\
\hline CESp.12 & $0.16-0.36$ & 0.39 & 0.43 & \\
\hline
\end{tabular}




\begin{tabular}{|c|c|c|c|c|}
\hline ITEM-FACTOR & INTER-ITEM & ITEM-FACTOR & ITEM-TOTAL & ALFA \\
\hline CESp.15 & $0.28-0.49$ & 0.59 & 0.47 & \\
\hline CESp.23 & $0.15-0.49$ & 0.55 & 0.40 & \\
\hline estratégia de jogo & $0.11-0.51$ & & & 0.72 \\
\hline CESp.02 & $0.11-0.51$ & 0.36 & 0.33 & \\
\hline CESp.04 & $0.20-0.40$ & 0.49 & 0.54 & \\
\hline CESp.08 & $0.16-0.40$ & 0.38 & 0.28 & \\
\hline CESp.09 & $0.19-0.51$ & 0.47 & 0.45 & \\
\hline CESp.11 & $0.16-0.37$ & 0.44 & 0.49 & \\
\hline CESp.17 & $0.11-0.36$ & 0.38 & 0.43 & \\
\hline CESp.21 & $0.20-0.40$ & 0.50 & 0.45 & \\
\hline técnica & $0.16-0.49$ & & & 0.73 \\
\hline CESp.07 & $0.16-0.49$ & 0.50 & 0.54 & \\
\hline CESp.14 & $0.16-0.29$ & 0.36 & 0.48 & \\
\hline CESp.16 & $0.26-0.43$ & 0.51 & 0.57 & \\
\hline CESp.18 & $0.18-0.40$ & 0.48 & 0.55 & \\
\hline CESp.20 & $0.18-0.34$ & 0.36 & 0.43 & \\
\hline CESp.22 & $0.28-0.49$ & 0.58 & 0.62 & \\
\hline construção do carácter & $0.37-0.67$ & & & 0.79 \\
\hline CESp.05 & $0.37-0.45$ & 0.48 & 0.52 & \\
\hline CESp.13 & $0.45-0.65$ & 0.71 & 0.60 & \\
\hline CESp.19 & $0.37-0.67$ & 0.63 & 0.55 & \\
\hline CESp.24 & $0.39-0.54$ & 0.57 & 0.53 & \\
\hline
\end{tabular}

Em termos gerais, estes valores convergem com os reportados noutros estudos realizados com a versão original (ainda que no estudo original os valores tenham sido relativamente superiores, variando entre 0.88 e 0.94) e fornecem suporte para a assunção da fiabilidade da CESp, tanto enquanto instrumento de avaliação multidimensional como unidimensional da eficácia do treinador, na linha portanto do sugerido por outros autores (e.g., 3, 15, 16, 36, 39, 41).

No mesmo sentido concorreu a análise das matrizes das correlações inter-item e item-total relativas a cada um dos 4 factores, verificando-se que a saturação dos itens no factor em que era suposto pertencerem foi, em todos os casos, positiva e apresentando magnitude moderada a forte. De uma forma geral, foi no factor 'carácter' que se verificaram as correlações com magnitude mais elevada, tanto entre itens como entre cada item e o respectivo factor. Ao invés, em qualquer dos outros três factores foi possível identificar algumas correlações relativamente reduzidas entre os vários itens que os constituíam, com valores 
compreendidos entre os 0.10 e os 0.20 , com dois itens (itens 3 e 6 , ambos integrando o factor 'motivação') a apresentarem mesmo valores inferiores, revelando portanto a existência de reduzida variância partilhada entre eles. Em todo o caso, de uma forma geral, todas as correlações se situaram dentro do intervalo de 0.15 - 0.50 proposto por Clark e Watson ${ }^{(12)}$.

Adicionalmente, todas as correlações dos itens com os respectivos factores apresentaram uma magnitude igual ou superior a 0.30 (com excepção do caso do item 3 , cujo valor ainda assim foi de 0.29), chegando, no caso do item 13 (pertencente ao factor 'carácter'), a ser mesmo superior a 0.70 , ilustrando portanto uma clara relação com o respectivo factor.

No quadro 3 apresentam-se os resultados referentes às correlações entre os quatro factores, bem como entre cada um dos factores e o total da escala, sendo possível verificar que todas elas foram positivas, com as primeiras (i.e., entre os quatro factores) a variarem entre 0.48 e 0.67 e as segundas (i.e., entre cada factor e a escala total), relativamente mais elevadas, a variarem entre 0.76 e 0.87 .

QUADRO 3 - Valores das correlações inter-factores e factor-total.

\begin{tabular}{|c|c|c|c|c|c|}
\hline FACTOR & 1 & 2 & 3 & 4 & TOTAL \\
\hline 1. Motivação & 1.00 & & & & 0.87 \\
\hline 2. Estratégia de jogo & 0.59 & 1.00 & & & 0.84 \\
\hline 3. Técnica & 0.65 & 0.66 & 1.00 & & 0.87 \\
\hline 4. Construção do carácter & 0.67 & 0.48 & 0.58 & 1.00 & 0.76 \\
\hline
\end{tabular}

Consistentemente com o que tem sido referido na literatura ${ }^{(12,27,33)}$, foi evidente a existência de correlações positivas entre os quatro factores. De salientar, porém, que, considerando que a magnitude das correlações entre os vários factores foi, em todos os casos, inferior a 0.70 , a sua independência e validade discriminante não parece estar em causa, diferenciando-se bem entre si e permitindo assim contribuir para uma representação mais válida do construto da eficácia do treinador. Quanto às correlações entre os factores e o valor total da escala, os valores são todos muito elevados, próximos de 1.00 , reflectindo assim uma estreita relação entre cada um dos factores representantes de diferentes facetas da eficácia do treinador e a eficácia total.

Assim sendo, os resultados deste estudo foram uma vez mais consistentes com os encontrados noutros estudos em que foram analisadas as propriedades psicométricas da versão original (e.g., 3,15,16,41) (ver quadro 4), situando-se entre os valores mais baixos e mais altos reportados, tanto para as correlações inter-factor como para as factor-total, na linha do já referido para os coeficientes de alfa de Cronbach. 


\begin{tabular}{llcc}
\hline ESTUDO & ALFA & INTER-FACTOR & ITEM-TOTAL \\
\hline Feltz et al. ${ }^{(15)}$ & 0.95 & $0.37-0.69$ & $0.68-0.86$ \\
\hline Tsorbatzudis et. al. ${ }^{(41)}$ & 0.82 & $0.42-0.62$ & $0.72-0.85$ \\
\hline Boardley et al. ${ }^{(3)}$ & 0.97 & $0.69-0.72$ & $0.86-0.93$ \\
\hline Feltz et al. ${ }^{(16)}$ & 0.95 & $0.40-0.76$ & $0.68-0.83$ \\
\hline
\end{tabular}

Tal como anteriormente mencionado, Feltz e colaboradores ${ }^{(15)}$, considerando as sugestões de Bandura (2) de que, embora os julgamentos de auto-eficácia tenham em conta especificidades e exigências contextuais e das tarefas a realizar, é igualmente importante considerar a existência de uma noção global de eficácia em função da natureza do domínio e do tipo de rendimento que se pretende predizer, conceptualizaram a eficácia do treinador de forma multidimensional mas também unidimensional, admitindo em conformidade a possibilidade de a CES ter uma estrutura multidimensional mas também unidimensional.

Nesse sentido, no presente estudo, foram avaliados dois modelos de medida distintos para a CESp. 0 modelo multidimensional, designado de $\mathrm{M}^{4}$, foi definido de acordo com os resultados reportados na literatura para a versão original, tanto em relação ao número de factores (i.e., 4) como aos itens que os constituem, assumindo a seguinte composição: i) Factor 1, designado 'motivação' e constituído pelos itens 1, 3, 6, 10, 12, 15 e 23; ii) Factor 2, designado 'estratégia de jogo' e constituído pelos itens 2, 4, 8, 9, 11, 17 e 21; iii) Factor 3, designado 'técnica' e constituído pelos itens 7, 14, 16, 18, 20 e 22; e iv) Factor 4, designado 'construção do carácter' e constituído pelos itens 5, 13, 19 e 24. Quanto ao modelo unidimensional, designado de $\mathrm{M}^{1}$, foi naturalmente composto por apenas um factor, designado de 'eficácia total' e constituído por todos os 24 itens da CESp.

Para a realização das AFC foi utilizada a matriz de covariância dos dados e adoptado o método da máxima verosimilhança, considerando a inexistência de indícios de violação da normalidade multivariada das respostas e as vantagens fornecidas por este procedimento de estimação ${ }^{(v e r 19)}$.

Normalmente, o primeiro indicador de ajustamento global do modelo inspeccionado a considerar é o $\chi^{2}$, ou seja a função da diferença entre a matriz de covariância dos dados analisados e a matriz do modelo postulado, correspondendo um valor elevado e estatisticamente significativo à existência de problemas de ajustamento do modelo ${ }^{(7,25)}$.

Todavia, é bem conhecida a sensibilidade desta estatística, por exemplo, à dimensão da amostra, pelo que, mesmo quando o valor de $\chi^{2}$ é elevado e estatisticamente significativo, se 
recomenda atender aos valores de outros indicadores que reflectem aspectos distintos do ajustamento global dos modelos em análise, como sejam o CFI, o NNFI, o RMSEA e a SRMR.

Adicionalmente, alguns autores recomendam igualmente atender à razão entre o valor do $\chi^{2}$ e os graus de liberdade, como um indicador ad hoc de ajustamento do modelo, destacando valores inferiores a 3 como reflexo de um bom ajustamento do modelo ${ }^{(24)}$.

Em conformidade, os resultados encontrados para ambos os modelos examinados (ver quadro 5), apesar dos já esperados valores elevados e estatisticamente significativos do $\chi^{2}$, sugerem a existência de um ajustamento aceitável à matriz de dados estudada, apontando no sentido da validade de ambas as estruturas factoriais testadas.

QUADRO 5 - Valores dos índices de bondade do ajustamento global dos modelos $\mathrm{M}^{1}$ e $\mathrm{M}^{4}$.

\begin{tabular}{lccccccc}
\hline MODELO & $\chi^{2}$ & gl & $\chi^{2} /$ gl & CFI & NNFI & SRMR & RMSEA (90\% IC) \\
\hline$M^{1}$ & $744.40 p<0,001)$ & 252 & 2.95 & 0.92 & 0.91 & 0.07 & $0.09(0.08-0.10)$ \\
\hline$M^{4}$ & $641.40 p<0.001)$ & 246 & 2.61 & 0.93 & 0.93 & 0.07 & $0.08(0.07-0.09)$ \\
\hline
\end{tabular}

Na verdade, em ambos os casos, os valores referentes ao CFI e ao NNFI, que representam as medidas das variâncias e covariâncias dos modelos e variam entre 0.00 e 1.00, foram superiores ao valor de corte de 0.90 , sugerido por vários autores ${ }^{(e .9 ., 11,32)}$ para este efeito, com o $\mathrm{M}^{4}$ a aproximar-se inclusivamente das sugestões mais exigentes de Hu e Bentler ${ }^{(23)}$, que propuseram um valor de corte de 0.95 para se classificar um modelo como aceitável.

Também os valores relativos ao RMSEA e à SRMR apontaram no sentido da demonstração da qualidade dos modelos examinados: enquanto o RMSEA se situou dentro dos valores de referência sugeridos pela literatura, que propõe a exclusão apenas de valores superiores a $0.10^{(5,7,28,31)}$, entendendo os iguais ou inferiores a 0.08 como reflexo de um ajustamento aceitável, a SRMR foi apenas ligeiramente superior ao valor de referência de 0.05 também sugerido na literatura da especialidade como o valor de corte a considerar para este efeito ${ }^{(7,25)}$.

Ainda assim, recorrendo às sugestões fornecidas pelo software utilizado, bem como à análise dos resultados anteriormente referidos, decidiu-se verificar se a partir da reespecificação de alguns dos seus parâmetros, designadamente no que se refere a permitir a existência de erros correlacionados entre alguns dos seus itens, seria possível melhorar os índices de ajustamento dos modelos examinados, o que veio efectivamente a suceder, tanto num caso como no outro (ver quadro 6). 
QUADRO 6 - Valores dos índices de bondade do ajustamento global dos modelos $M^{1}$ e $M^{4}$ reespecificados.

\begin{tabular}{lccccccc}
\hline MODELO & $\chi^{2}$ & gl & $\chi^{2} / g l$ & CFI & NNFI & SRMR & RMSEA (90\% IC) \\
\hline$M^{1 a}$ & $679.89 P<0.001)$ & 251 & 2.71 & 0.93 & 0.92 & 0.07 & $0.08(0.08-0.09)$ \\
\hline$M^{1 b}$ & $649.00 P<0.001)$ & 250 & 2.60 & 0.93 & 0.93 & 0.07 & $0.08(0.07-0.09)$ \\
\hline$M^{4 a}$ & $590.89 P<0,001)$ & 245 & 2.41 & 0.94 & 0.93 & 0.06 & $0.08(0.07-0.08)$ \\
\hline$M^{4 b}$ & $569.91 P<0.001)$ & 244 & 2.33 & 0.95 & 0.94 & 0.06 & $0.07(0.06-0.08)$ \\
\hline
\end{tabular}

Nota: $M^{1 a}$ (erros correlacionados entre itens 2-9); $M^{2 b}$ (erros correlacionados entre itens 2-9 e 23-24); $M^{4 a}$ (erros correlacionados entre itens 2-9); e M ${ }^{4 b}$ (erros correlacionados entre itens 2-9 e 23-24).

Os pesos factoriais e os respectivos erros padrão, os valores te a magnitude da variância que cada item partilha com o respectivo factor, no caso do $\mathrm{M}^{4}$, estão apresentados no quadro 7.

QUADRO 7 - Estimativas do peso factorial (erro padrão), valor t e variância que oS factores extraem dos itens da CESp, quando considerado o $\mathrm{M}^{4}$.

\begin{tabular}{|c|c|c|c|c|c|c|}
\hline ITEM & MOTIVAÇÃO & $\begin{array}{c}\text { ESTRATÉGIA } \\
\text { DE JOGO }\end{array}$ & TÉCNICA & $\begin{array}{l}\text { CONSTRUÇÃO } \\
\text { DO CARÁCTER }\end{array}$ & VALOR T & $\mathrm{R}^{2}$ \\
\hline CESp.01 & $0.33(0.04)$ & & & & 8.76 & 0.11 \\
\hline CESp.02 & $0.31(0.04)$ & & & & 7.08 & 0.10 \\
\hline CESp.03 & $0.31(0.04)$ & & & & 7.48 & 0.10 \\
\hline CESp.04 & $0.33(0.04)$ & & & & 9.01 & 0.11 \\
\hline CESp.05 & $0.31(0.04)$ & & & & 7.79 & 0.10 \\
\hline CESp.06 & $0.42(0.04)$ & & & & 10.88 & 0.18 \\
\hline CESp.07 & $0.36(0.04)$ & & & & 9.69 & 0.13 \\
\hline CESp.08 & & $0.27(0.05)$ & & & 5.50 & 0.07 \\
\hline CESp.09 & & $0.35(0.04)$ & & & 7.85 & 0.12 \\
\hline CESp.10 & & $0.46(0.04)$ & & & 10.97 & 0.21 \\
\hline CESp.11 & & $0.38(0.05)$ & & & 8.00 & 0.14 \\
\hline CESp.12 & & $0.35(0.04)$ & & & 8.48 & 0.12 \\
\hline CESp.13 & & $0.42(0.04)$ & & & 9.98 & 0.18 \\
\hline CESp.14 & & $0.45(0.04)$ & & & 10.58 & 0.20 \\
\hline CESp.15 & & & $0.35(0.05)$ & & 7.18 & 0.12 \\
\hline CESp.16 & & & $0.40(0.05)$ & & 8.42 & 0.16 \\
\hline
\end{tabular}




\begin{tabular}{|c|c|c|c|c|c|c|}
\hline ITEM & MOTIVAÇÃO & $\begin{array}{c}\text { ESTRATÉGIA } \\
\text { DE JOGO }\end{array}$ & TÉCNICA & $\begin{array}{l}\text { CONSTRUÇÃO } \\
\text { DO CARÁCTER }\end{array}$ & VALOR T & $\mathrm{R}^{2}$ \\
\hline CESp.17 & & & $0.39(0.04)$ & & 10.10 & 0.15 \\
\hline CESp.18 & & & $0.51(0.04)$ & & 11.97 & 0.26 \\
\hline CESp.19 & & & $0.38(0.04)$ & & 8.71 & 0.14 \\
\hline CESp.20 & & & $0.43(0.04)$ & & 10.84 & 0.18 \\
\hline CESp.21 & & & & $0.40(0.04)$ & 9.43 & 0.16 \\
\hline CESp.22 & & & & $0.53(0.05)$ & 10.70 & 0.28 \\
\hline CESp.23 & & & & $0.50(0.04)$ & 11.19 & 0.25 \\
\hline CESp.24 & & & & $0.44(0.04)$ & 10.46 & 0.19 \\
\hline
\end{tabular}

De uma forma geral, foi evidente que os valores de saturação no respectivo factor não foram muito elevados, variando entre 0.30 (com excepção do item 2, cujo peso factorial foi de 0.27 ) e 0.53 , razão pela qual também foram relativamente reduzidos os valores da magnitude da variância atribuída ao factor correspondente, os quais (com a excepção do item 2, cujo valor foi de 0.07 ) variaram entre 0.10 e 0.28 . Os itens $15,8,18$ e 13 foram os itens que melhor descreveram os factores 1, 2, 3 e 4, respectivamente. Quanto aos valores $t$, foram todos relativamente elevados e estatisticamente significativos.

Do mesmo modo, também em relação ao $\mathrm{M}^{1}$ (ver quadro 8 ), foi possível verificar que os valores de saturação no factor único não foram muito elevados, variando entre 0.23 , para o item 2, e 0.51, para o item 18, que foi o item que melhor descreveu o factor; em conformidade, também os valores da magnitude da variância atribuída ao factor foram reduzidos, variando entre 0.05 e 0.26 . Quanto aos valores $t$, foi evidente que foram todos relativamente elevados e estatisticamente significativos.

QUADRO 8 - Estimativas do peso factorial (erro padrão), valor t e variância que os

factores extraem dos itens da CESp, quando considerado o $\mathrm{M}^{1}$.

\begin{tabular}{llll}
\hline ITEM & EFICÁCIA & VALOR T & $\mathrm{R}^{2}$ \\
\hline CESp.01 & $0.32(0.04)$ & 8.64 & 0.10 \\
\hline CESp.02 & $0.23(0.05)$ & 4.73 & 0.05 \\
\hline CESp.03 & $0.31(0.04)$ & 7.13 & 0.10 \\
\hline CESp.04 & $0.32(0.04)$ & 7.27 & 0.10 \\
\hline CESp.05 & $0.37(0.04)$ & 8.92 & 0.14 \\
\hline CESp.06 & $0.33(0.04)$ & 7.96 & 0.11 \\
\hline CESp.07 & $0.33(0.05)$ & 7.07 & 0.11 \\
\hline
\end{tabular}




\begin{tabular}{|c|c|c|c|}
\hline ITEM & EFICÁCIA & VALOR T & $\mathrm{R}^{2}$ \\
\hline CESp.08 & $0.41(0.04)$ & 9.84 & 0.17 \\
\hline CESp.09 & $0.32(0.05)$ & 6.75 & 0.10 \\
\hline CESp.10 & $0.32(0.04)$ & 8.73 & 0.10 \\
\hline CESp.11 & $0.31(0.04)$ & 8.04 & 0.10 \\
\hline CESp.12 & $0.29(0.04)$ & 7.32 & 0.08 \\
\hline CESp.13 & $0.43(0.05)$ & 8.72 & 0.18 \\
\hline CESp.14 & $0.37(0.05)$ & 8.04 & 0.14 \\
\hline CESp.15 & $0.40(0.04)$ & 10.55 & 0.16 \\
\hline CESp.16 & $0.37(0.04)$ & 9.78 & 0.14 \\
\hline CESp.17 & $0.40(0.04)$ & 9.68 & 0.16 \\
\hline CESp.18 & $0.50(0.04)$ & 11.82 & 0.26 \\
\hline CESp.19 & $0.43(0.04)$ & 10.01 & 0.18 \\
\hline CESp.20 & $0.37(0.04)$ & 8.61 & 0.14 \\
\hline CESp.21 & $0.40(0.04)$ & 9.66 & 0.16 \\
\hline CESp.22 & $0.42(0.04)$ & 10.85 & 0.18 \\
\hline CESp.23 & $0.36(0.04)$ & 9.91 & 0.13 \\
\hline CESp.24 & $0.41(0.04)$ & 10.05 & 0.17 \\
\hline
\end{tabular}

Ao compararmos os resultados da AFC ao modelo de medida multidimensional do presente estudo com os disponíveis na literatura relativamente à bondade do ajustamento da mesma estrutura factorial às matrizes de dados recolhidas noutros estudos através da versão original da CES (ver quadro 9), é possível constatar que a CESp se destaca positivamente.

QUADRO 9 - Valores dos índices de bondade do ajustamento global da estrutura tetra-factorial da versão original da CES reportados noutros estudos.

\begin{tabular}{|c|c|c|c|c|c|}
\hline ESTUDO & S-B $\chi^{2}$ & $\mathrm{~S}-\mathrm{B} \chi^{2} / \mathrm{gl}$ & CFI & RMSEA & SRMR \\
\hline Feltz et al. ${ }^{(15)}$ & 790.21 & 3.21 & 0.89 & 0.08 & n.d. \\
\hline Sullivan \& Kent ${ }^{(39)}$ & n.d. & n.d. & 0.87 & 0.09 & 0.09 \\
\hline Tsorbatzudis et. al. (41) & 258.71 & 1.14 & 0.92 & 0.07 & 0.07 \\
\hline Tsorbatzudis et. al. (41) a & 130.01 & 0.93 & 0.96 & 0.05 & 0.05 \\
\hline Myers et al. (36) b & 1549.86 & 6.30 & 0.92 & 0.10 & 0.04 \\
\hline Kavussanu et al (26) & 595.32 & 2.42 & 0.90 & 0.07 & 0.07 \\
\hline
\end{tabular}




\begin{tabular}{lccccc}
\hline ESTUDO & S-B $\chi^{2}$ & S-B $\chi^{2} / g l$ & CFI & RMSEA & SRMR \\
\hline Boardley et al. $^{(3)}$ & 419.74 & 1.71 & 0.87 & 0.07 & 0.07 \\
\hline Boardley et al. $^{(3)}{ }^{(3)}$ & 348.45 & 1.56 & 0.90 & 0.06 & 0.05 \\
\hline Feltz et al. (16) $^{(16)}$ & 901.42 & 3.66 & 0.97 & 0.08 & n.d. \\
\hline
\end{tabular}

${ }^{a}$ CES apenas com 20 itens, tendo sido removidos os itens 6, 8, 9 e 12. ${ }^{\text {b }}$ CES foi utilizada para avaliar a competência dos treinadores e não a sua eficácia; ' CES apenas com 23 itens, tendo sido removido o item 3. n.d.= não disponível.

$\mathrm{Na}$ verdade, se nos concentrarmos, por exemplo, nos valores relativos ao CFI verificamos que, exceptuando Feltz e colaboradores ${ }^{(16)}$, em todos os outros estudos o valor encontrado foi inferior ao do presente estudo, com ou sem reespecificação. E, no mesmo sentido, também os valores encontrados no presente estudo para o RMSEA e a SRMR estão entre os que reflectem um maior ajustamento do modelo de medida postulado à matriz de covariância examinada.

Conforme anteriormente verificado, os indicadores de ajustamento encontrados para o modelo de medida unidimensional neste estudo não foram tão bons como os registados para o modelo multidimensional, na linha aliás do referido na literatura ${ }^{(36)}$. Todavia, ainda assim, quando comparados, por exemplo, com os reportados pelos autores da versão original (i.e., $\chi^{2}=844$; NNFI=0.87; $C F I=0.88$; e RMSEA=0.09), ou mesmo com os reportados por Tsorbatzudis e colaboradores ${ }^{(41)}$ (i.e., $\chi^{2}=243.25$; NNFI=0.91; CFI=0.93; SRMR=0.06; e RMSEA=0.07; ou $\chi^{2}=227$; NNFI=0.92; CFI=0.94; e SRMR=0.06; e RMSEA=0.07, com a exclusão dos itens 6, 8, 9 e 12) concluímos no sentido da existência de um ajustamento aceitável do modelo avaliado no presente estudo.

\section{CONCLUSÕES}

De uma forma geral e consistente, os resultados encontrados neste estudo revelam a qualidade das propriedades psicométricas e da estrutura factorial da CESp, suportando portanto a recomendação da sua utilização pelos interessados em investigar o modo como os treinadores avaliam a sua eficácia na realização de um alargado conjunto de tarefas.

Adicionalmente, não obstante a CES ter sido essencialmente desenhada como um instrumento multidimensional para avaliar a eficácia do treinador desportivo, as evidências recolhidas neste estudo apontam no sentido de ela poder considerar-se igualmente como um instrumento unidimensional, fornecendo assim um valor único de eficácia do treinador. De resto, esta possibilidade não só encontra eco no que tem vindo a ser efectuado por outros investigadores ${ }^{(e .9,15)}$ como se justifica conceptualmente ${ }^{(2)}$. 
Na realidade, importará destacar que os resultados das AFC realizadas à CESp expressaram que, em qualquer dos dois casos, os valores dos diferentes indicadores da qualidade de ajustamento dos modelos de medida inspeccionados forneceram suporte para a recomendação da sua utilização pelos investigadores nacionais, os quais poderão, portanto, utilizá-la com confiança tanto como uma medida global para a avaliação da eficácia dos treinadores como para avaliar individualmente cada uma das quatro facetas nela representadas.

Ainda assim, apesar de os resultados encontrados apontarem no sentido da fiabilidade e validade da versão portuguesa da CES, convirá destacar a necessidade de serem desenvolvidos mais estudos no sentido de garantir a plausibilidade dos modelos de medida testados, designadamente através da realização de estudos que verifiquem a sua invariância configuracional quando aplicada com amostras com características distintas da considerada neste estudo. 
1. Bandura A (1977). Self-efficacy: Toward a unifying theory of behavior change. Psychological Review, 84 (2): 191-215.

2. Bandura A (1997). Self-efficacy: the exercise of control. New York: Freeman.

3. Boardley I, Kavussanu M, Ring C (2008). Athletes' perceptions of coaching effectiveness and athlete-related outcomes in Rugby Union: An investigation based on the coaching efficacy model. The Sport Psychologist, 22: 269-287.

4. Borrie A (1996). Coaching science. In: Reilly T(ed), Science and soccer. London: E \& F N Spon, 243-258.

5. Browne MW, Cudeck R (1993). Alternative ways of assessing model fit. In: Bollen KA, Long JS (eds), Testing structural equation models. Newbury Park, CA: Sage Publications, 136-162.

6. Buceta JM (1996). Psicologia del Deporte Aplicada: Áreas de intervención en relación con el entrenador. In: Cruz JF (ed), Manual de psicologia do desporto. Braga: Sistemas Humanos e Organizacionais, 673-679.

7. Byrne BM (1998). Structural equation modeling with LISREL, PRELIS and SIMPLIS: Basic concepts, applications and programming. Mahwah, NJ: Lawrence Erlbaum Associates.

8. Campbell T, Sullivan P (2005). The effect of a standardized coaching education program on the efficacy of novice coaches. Avante, 11 (1): 38-45.

9. Chase MA, Lirgg CD, Feltz DL (1997). Do coaches' efficacy expectations for their teams predict team performance? The Sport Psychologist, 11: 8-23.

10. Chelladurai P, Saleh SD (1980). Dimensions of leader behavior in sports: Development of a leadership scale. Journal of Psychology, 2: 34-45.

11. Chi, L. \& Duda, J. L. (1995). Multi-sample confirmatory factor analysis of the Task and Ego Orientation in Sport Questionnaire. Research Quarterly for Exercice and Sport, 66 (2), 91-98.

12. Clark LA, Watson D (1995). Constructing validity: Basic issues in objective scale development. Psychological Assessment, 7: (3), 309-319.
13. Denham CH, Michael JJ (1981). Teacher sense of efficacy: A definition of the construct and a model for further research. Educational Research Quarterly for Exercise and Sport, 5: 39-63.

14. Feltz DL, Chase MA (1998). The measurement of self-efficacy and confidence in sport. In: Duda JL (ed), Advancements in sport and exercise psychology measurement. Morgantown, WV: Fitness Information Technology, 63-78.

15. Feltz DL, Chase MA, Moritz SE, Sullivan PJ (1999). A conceptual model of coaching efficacy: Preliminary investigation and instrument development. Journal of Educational Psychology, 91: 765-776.

16. Feltz DL, Hepler T, Roman N, Paiement C, (2009). Coaching efficacy and volunteer youth sport coaches. The Sport Psychologist, 23: 24-41.

17. Fonseca AM, Rocha H (1995). Perception par les coaches du style de leadership préféré des athlètes. Sport, 38 (3): 151, 40-45.

18. Fung $L$ (2003). Assesment: Coaching efficacy as indicators of coach education program needs. Athletic Insigth, Online Journal of Psychology, 5: 12-18.

19. Harrington D (2009). Confirmatory factor analysis. New York: Oxford University Press.

20. Hedstrom RA (2006). The developing coach: A season-long investigation of coaching efficacy and coaching competency, feedback, and practice behaviors. Tese de doutoramento não publicada. Michigan State University. 21. Horn TS (2002). Coaching effectiveness in the sports domain. In: Horn TS (ed), Advances in sport psychology. Champaign, IL: Human Kinetics, 309-354. 22. Houllier G, Crevoisier J (1993). Entraîneur competence et passion, les details qui font gagner. France: Canal + Èditions.

23. Hu L, Bentler PM (1999). Cutoff criteria for fit indexes in covariance structure analysis: Conventional criteria versus new alternatives. Structural Equation Modeling, 6 (1): 1-55.

24. Joreskog KG (1969). A general approach to confirmatory maximum likehood factor analysis. Psychometrica, 34: 183-22. 
25. Joreskog KG, Sorbom D (1989). LISREL 7: A guide to the program and applications. Chicago, IL: Scientific Software International Inc.

26. Kavussanu M, Boardley D, Jutkiewicz N, Vincent S, Ring $C$ (2008). Coaching efficacy and coaching effectiveness: Examining their predictors and comparing coaches and athletes reports. The Sport Psychologist, 22: 383-404.

27. Kent A, Sullivan PJ (2003). Coaching efficacy as a predictor of university coaches' commitment. International Sports Journal, Winter: 78-87.

28. Kline RB (2005), Principles and practice of structural equation modeling (2nd edition). New York: The Guilford Press.

29. Kowalski C, Edginton C, Lankford S, Waldron J, Roberts-Dobie S, Nielsen L (2007). Coaching efficacy and volunteer youth soccer coaches. Asian Journal of Exercise \& Sports Science, 4 (1): 1-5.

30. Lyle J (2002). Sport coaching concepts. A framework for coaches behaviour. New York: Routledge.

31. MacCallum RC, Browne MW, Sugawara HM (1996). Power analysis and determination of sample size for covariance structure modeling. Psychological Methods, 1: 130-149.

32. Maia JA (1996). Um discurso metodológico em torno da vaidade do construto: Posições de um lisrelita. In: Almeida L, Araújo S, Gonçalves MM, Machado C, Simões MR (eds), Avaliação psicológica: Formas e contextos. APPORT, Associação dos Psicólogos Portugueses, 43-59.

33. Malete L, Feltz D (2000). The effect of a coaching education program on coaching efficacy. The Sport Psychologist, 14: 410-417.

34. Myers ND, Feltz DL, Chase MA, Reckase MD, Hancock DR (2008). The Coaching Efficacy Scale II High school teams. Educational and Psychological Measurement, 68: 1059-1076.

35. Myers ND, Feltz DL, Maier KS, Wolfe EW, Reckase MD (2006). Athletes' evaluations of their head coach's coaching competency. Research Quarterly for Exercise and Sport, 77: 111-121.
36. Myers ND, Wolfe E, Feltz D (2005). An evaluation of the psychometric properties of the Coaching Efficacy Scale for coaches from the United States of America. Measurement in Physical Education and Exercice Science, 9 (3) : 135-160.

37. Park JK (1992). Construction of the Coaching Confidence Scale. Tese de doutoramento não publicada. Michigan State University.

38. Ramirez MCP (2002). Caracterización del entrenador de Alto Rendimento Deportivo. Cuadernos de Psicologia del Deporte, 2: 16-37.

39. Sullivan P, Kent A (2003). Coaching efficacy as a predictor of leadership style in intercollegiate athletics. Journal of Applied Sport Psychology, 15: 1-11.

40. Thelwell RC, Lane AM, Weston NJV, Greenlees IA (2008). Examining relationships between emotional intelligence and coaching efficacy. International Journal of Sport and Exercise Psychology, 6: 224-235. 41. Tsorbatzudis H, Daroglou G, Zahariadis P, Grouious $G$ (2003). Examination of coaches self-efficacy preliminary analysis of the Coaching Efficacy Scale. Perceptual and Motor Skills, 97: 1297-1306.

42. Vargas-Tonsing T, Warners A, Feltz DL (2003). The predictability of coaching efficacy on team efficacy and player efficacy in Volleyball. Journal of Sport Behavior, 26 (4): 396-407.

43. Whitmore J (1994). Coaching for performance. A practical guide to growing your own skills. London: Nicholas Brealey Publishing 